\title{
A Human Vascular Model Based on Microdialysis for the Assessment of the Vasoconstrictive Dose-Response Effects of Norepinephrine and Vasopressin in Skin
}

Kim Tchou Folkesson, Anders Samuelsson, Erik Tesselaar, Bengt Dahlström and Folke Sjöberg

\section{Linköping University Post Print}

N.B.: When citing this work, cite the original article.

This is the authors' version of the final publication:

Kim Tchou Folkesson, Anders Samuelsson, Erik Tesselaar, Bengt Dahlström and Folke Sjöberg, A Human Vascular Model Based on Microdialysis for the Assessment of the Vasoconstrictive Dose-Response Effects of Norepinephrine and Vasopressin in Skin, 2012, Microcirculation, (19), 4, 352-359.

http://dx.doi.org/10.1111/j.1549-8719.2012.00170.x

Copyright: Informa Healthcare / John Wiley and Sons http://www.wiley.com/

Postprint available at: Linköping University Electronic Press http://urn.kb.se/resolve?urn=urn:nbn:se:liu:diva-77530 


\section{A human vascular model based on microdialysis for the assessment of the vasoconstrictive dose response effects of noradrenaline and vasopressin in skin}

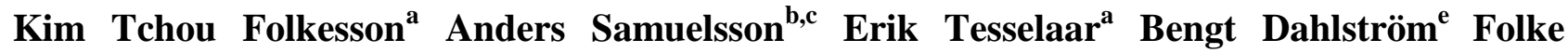
Sjöberg ${ }^{\text {a,c,d }}$

Departments of ${ }^{\mathrm{a}} \mathrm{Clinical}$ and Experimental Medicine and ${ }^{\mathrm{b}}$ Medicine and Health Sciences, Faculty of Health Sciences, Linköping University, Linköping, Sweden. ${ }^{c}$ Department of Intensive Care, ${ }^{\mathrm{d}}$ Hand and Plastic Surgery and Burns, University Hospital, Linköping, Sweden, ${ }^{\mathrm{e}}$ AB Biopharmacon, Uppsala, Sweden.

Corresponding author:

Prof. Folke Sjöberg

Departments of Anesthesiology and Clinical and Experimental Medicine

Linköping University Hospital

58185 Linköping, Sweden

E-mail: folke.sjoberg@liu.se

phone: +46101031820 Fax: +46101033685 


\section{Abstract}

Microdialysis enables drug delivery in the skin and simultaneous measurement of their effects. The present study aimed to evaluate whether increasing concentrations of noradrenaline (NA, 1.8-59 $\mu \mathrm{mol} / \mathrm{L}$ ) and vasopressin (VP, 1-100 nmol/L), delivered sequentially in one catheter or simultaneously through four catheters, yield dose-dependent changes in blood flow (as measured using urea clearance) and metabolism (glucose and lactate). We found a significant dose-dependent vasoconstriction with both drugs. Responses were characterized by a sigmoid dose-response model. Urea in the dialysate increased from a baseline of $7.9 \pm 1.7$ to $10.9 \pm 0.9 \mathrm{mmol} / \mathrm{L}$ for the highest concentration of NA $(\mathrm{p}<0.001)$ and from $8.1 \pm 1.4$ to $10.0 \pm 1.7 \mathrm{mmol} / \mathrm{L}$ for the highest concentration of VP ( $\mathrm{p}=0.037$ ). Glucose decreased from $2.3 \pm 0.7$ to $0.41 \pm 0.18 \mathrm{mmol} / \mathrm{L}$ for NA $(\mathrm{p}=0.001)$ and from $2.7 \pm 0.6$ to $1.3 \pm 0.5 \mathrm{mmol} / \mathrm{L}$ for $\mathrm{VP}(\mathrm{p}<0.001)$. Lactate increased from $1.1 \pm$ 0.4 to $2.6 \pm 0.5 \mathrm{mmol} / \mathrm{L}$ for NA $(\mathrm{p}=0.005)$ and from $1.1 \pm 0.4$ to $2.6 \pm 0.5 \mathrm{mmol} / \mathrm{L}$ for $\mathrm{VP}$ $(\mathrm{p}=0.008)$. There were no significant differences between responses from a single catheter and from those obtained simultaneously using multiple catheters. Microdialysis in the skin, either with a single catheter or using multiple catheters, offers a useful tool for studying dose response effects of vasoactive drugs on local blood flow and metabolism without inducing any systemic effects.

Keywords:

Microdialysis; Urea; Skin; Noradrenalin; Vasopressin; Dose Response; Pharmacodynamics; Human; Vasoconstriction 


\section{Introduction}

Much of our knowledge about the effects of drugs on microvascular function stems from dose response studies on isolated vessels and from intravital microscopic studies. These models have been of paramount importance in the development of new vasoactive drugs. An important shortcoming of in vitro models is the lack of interaction between mechanical and biochemical components of tissue cell function during, for example, vasoconstriction. Therefore, vasoconstriction effects in vitro cannot be extrapolated to clinical efficacy or outcome, which is why accurate and reproducible in vivo techniques to assess microvascular effects of vasoactive drugs in humans are needed.

Skin microdialysis is a minimally invasive technique, which has been used successfully during the last 20 years to measure substances in the extracellular space $(16,20,24)$. Besides this, microdialysis makes it possible to deliver drugs locally into tissues and at the same time measure their effects at the site of action (13).

When vasoactive drugs are delivered by microdialysis, their effect on local blood flow can be assessed if a marker substance is added to the microdialysis perfusate (18). This is because the diffusion of substances into the tissue is dependent on the local blood flow. With vasoconstriction, there is less diffusion of the marker into the tissue and therefore the concentration of the marker in the dialysate will be increased. Vasodilatation, likewise, results in lower marker concentrations in the dialysate. We have previously shown that urea is a suitable marker to measure blood flow during microdialysis at low flow rates and we have used the 'urea clearance' technique in muscle, liver and skin (8-10). The use of marker substances are particularly useful when it is not possible to use external optical techniques such a s laser-speckle contrast imaging (6) or laser-Doppler flowmetry (10).

Noradrenaline (NA) is a catecholamine that stimulates postsynaptic $\alpha$-adrenoceptors in arteries and veins to produce vasoconstriction, and the drug is widely used to maintain organ perfusion during shock. Vasopressin (VP) is an antidiuretic hormone that is increasingly used as an 
adjunct to NA and is thought to reverse some of the pathological vasodilatory processes produced by shock $(11,23)$. VP constricts mainly arterial blood vessels by binding to V1 receptors, which, similar to NA, causes vasoconstriction via the $\mathrm{IP}_{3}$ signal transduction pathway.

In the present study, our primary aim was to use the urea clearance technique to assess the vasoconstrictor responses to increasing concentrations of NA and VP in the skin of healthy volunteers. We hypothesized that we could in this way characterize dose response relations, as is commonly done in studies on isolated vessels. Our secondary aim was to investigate the metabolic effects of NA and VP in the skin by measuring glucose and lactate concentrations. Finally, we aimed to find out whether the results depended on how drugs were delivered: through a single catheter with increasing drug concentrations, or through multiple catheters, each with a different drug concentration.

\section{Methods}

\section{Subjects}

Twelve healthy subjects (6 males), mean age 23 (range 20-29) years, were included in the study. The subjects took no medication except for hormonal contraceptives, paracetamol up to $1 \mathrm{~g}$ four times daily, and nasal decongestants in the short term. They did not smoke tobacco or other nicotine-containing products, or consume coffee, tea, or alcoholic drinks for 72 hours before and during the study. Every subject received verbal and written information about the study and provided written informed consent. Participation was voluntary and subjects were free to withdraw at any time during the study without further explanation. The study conforms with the principles outlined in the Declaration of Helsinki and was approved by the regional ethics review board.

\section{Microdialysis}

The study was performed in a temperature-controlled room $\left(25 \pm 1{ }^{\circ} \mathrm{C}\right)$. During the study each volunteer rested in supine position. Two $20 \mathrm{kDa}$ microdialysis catheters (CMA 66, CMA 
Microdialysis AB, Solna, Sweden) were inserted in the skin of the volar side of each forearm. Care was taken to insert the catheter with little or no bleeding, and to avoid large vessels. Each catheter was inserted using a small needle introducer, which was withdrawn, leaving the catheter in place. Previous measurements with a DermaScan ultrasound device indicated that catheters are located on the boundary of dermis and hypodermis. The catheters were then fixed to the skin with transparent sterile plastic film.

Inserting microdialysis catheters in the skin causes a local trauma. To let any effects of this insertion trauma subside, a 60-minute recovery period was applied, during which the catheters were perfused with Ringer's acetate with urea $20 \mathrm{mmol} / \mathrm{L}$ (APL, Umeå, Sweden). This was done using precision pumps (CMA 107, CMA Microdialysis AB, Solna, Sweden) set at $0.5 \mu 1 /$ minute. After the recovery period, the subjects were randomly assigned to a treatment group. Six subjects were given NA (group 1), and the others were given VP (group 2). Both drugs were dissolved in Ringer's acetate with urea $20 \mathrm{mmol} / \mathrm{L}$ (Apoteket Farmaci, Linköping, Sweden) on the day of the experiments.

\section{Drug delivery}

The drug delivery protocol (Figure 1) started with a 45-minute baseline during which catheters were perfused at a flow rate of $0.5 \mu \mathrm{l} /$ minute with Ringer's acetate with urea $20 \mathrm{mmol} / \mathrm{L}$. Then, NA (group 1) or VP (group 2) were delivered simultaneously in all four catheters. In catheter 1, four increasing concentrations of NA $(1.8,5.9,18$ and $59 \mu \mathrm{mol} / \mathrm{L})$ or VP $(3.0,10,30$ and 100 nmol/L) were infused. The other catheters (2, 3 and 4) were used to deliver only the second, the third or the fourth drug concentration, respectively. In the first three subjects, NA concentrations were 100 times lower and VP concentrations 10 times lower. Since these concentrations did not elicit any effect, they were subsequently increased, but data from these subjects are included in the results. Each drug concentration was infused for 75 minutes. A flush period of five minutes was 
applied after every change in drug concentration at a perfusion flow rate of $15 \mu \mathrm{L} / \mathrm{min}$ to shorten the equilibrium times and to remove any air bubbles.

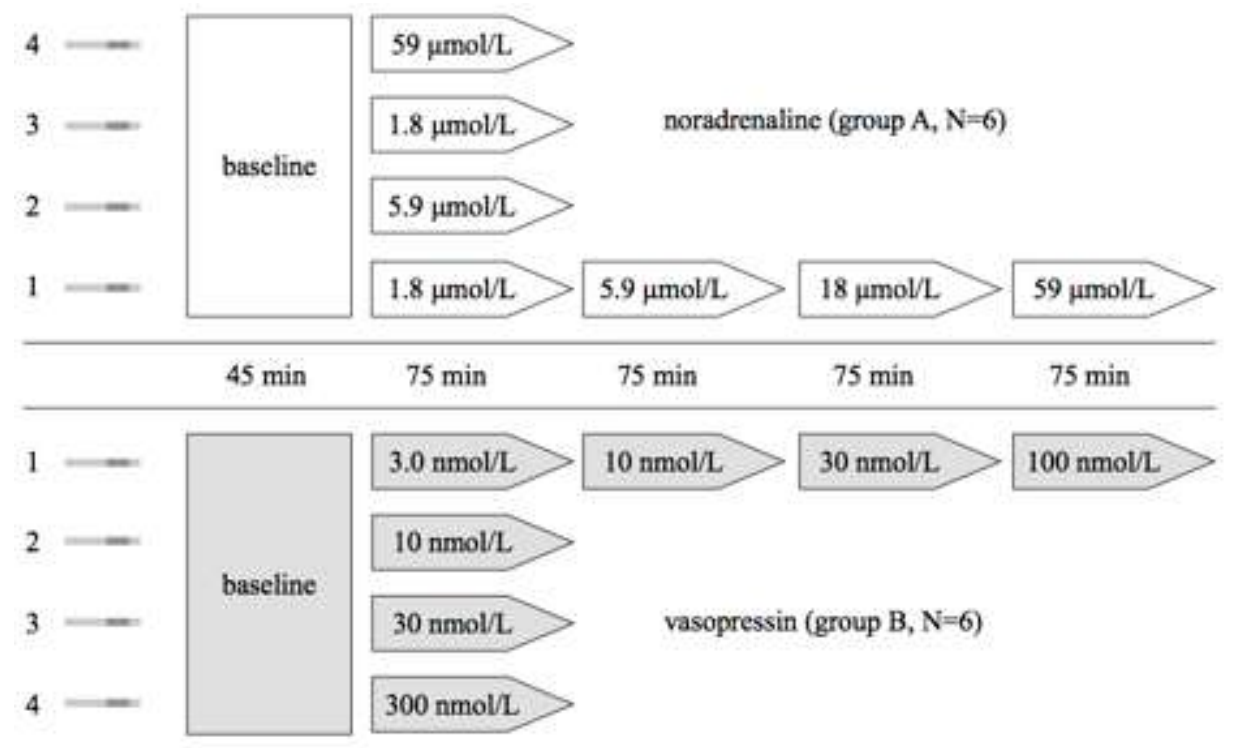

Figure 1. Dosage regimen. Subjects were randomly divided into two groups, $n=6$ in each group, and were given either noradrenaline or vasopressin. Each volunteer had a total of four microdialysis catheters inserted in the dermis of the forearm. After a 60 minute recovery period, each catheter was perfused with a different drug concentration. In catheter 1 , which was perfused with the lowest concentration, this was followed by three additional, increasing drug concentrations. The study took roughly 7 hours to conduct.

\section{Dialysate analysis}

The dialysate was collected in microvials that were exchanged every 15 minutes. The dialysate samples $(7.5 \mu \mathrm{L})$ were immediately analyzed for urea, glucose, and lactate using a chemical analyzer (CMA 600, CMA Microdialysis AB, Solna, Sweden). The analyzer uses enzymatic reagents and colorimetric measurements. Reagents were obtained from CMA Microdialysis AB (Solna, Sweden). The analyzer has an imprecision of $<3 \%$ for urea, $<5 \%$ for glucose, and $<6 \%$ for lactate. The linearity is $>95 \%$ for urea, glucose, and lactate.

\section{Data analysis and statistics}

Baseline concentrations of urea, glucose and lactate in the dialysate were calculated as the mean of the concentrations in the 3 samples collected during baseline. Dose responses were calculated as the mean of the last 4 samples of urea, glucose and lactate within each concentration (the first measurement after flushing of the catheter was discarded). A repeated measures analysis 
of variance (ANOVA) was performed to test for trends during baseline or within a concentration interval. Results are expressed as absolute change from baseline (mean $\pm \mathrm{SD}$ ). A sigmoid $\mathrm{E}_{\max }$ model (12) was fitted to the dose responses using non-linear regression. The model estimated the effective concentration that caused $50 \%$ of the vasoconstrictor response $\left(\mathrm{ED}_{50}\right)$. The sum of squares F-tests were used to test for differences between curves. Probabilities of less than 0.05 were accepted as significant. All statistical calculations were done with the help of GraphPad Prism version 5.00 for Windows (GraphPad Software, San Diego California USA, www.graphpad.com). 


\section{Results}

The introduction of the catheters was well-tolerated, with no notable reactions other than transient local erythema. There were no systemic inflammatory reactions attributable to microdialysis and no signs of infection at the site of implantation of the catheter up to 5 days after insertion. After the 60-minute recovery period after inserting the catheters, the baseline concentration of glucose and lactate were stable, while there was a slight but insignificant decrease in the concentration of urea (repeated measures ANOVA, $p=0.3$ ). The delivery of NA and VP resulted in a visible blanching of the skin surrounding the catheter in most of the volunteers.

A total of 568 samples of dialysate were obtained from 48 catheters in 12 healthy volunteers. Data points were missing from 11 catheters. In 8 catheters, this was because no dialysate fluid was collected at some point during the study. In 3 catheters, no baseline values could be measured.

After delivery of the highest concentration of NA, the urea concentration had increased by $38 \%$ from a baseline of $7.9 \pm 1.7 \mathrm{mmol} / \mathrm{L}$ to a final dialysate concentration of $10.9 \pm 0.9 \mathrm{mmol} / \mathrm{L}$. The glucose concentration decreased by $84 \%$ from $2.3 \pm 0.7$ to $0.41 \pm 0.18 \mathrm{mmol} / \mathrm{L}$ and lactate increased by $220 \%$ from $1.0 \pm 0.2$ to $3.2 \pm 0.8 \mathrm{mmol} / \mathrm{L}$. After the highest concentration of VP, the urea concentration had increased by $23 \%$ from $8.1 \pm 1.4$ to $10.0 \pm 1.7 \mathrm{mmol} / \mathrm{L}$, glucose decreased by $52 \%$ from $2.7 \pm 0.6$ to $1.3 \pm 0.5 \mathrm{mmol} / \mathrm{L}$ and lactate increased by $136 \%$ from $1.1 \pm 0.4$ to $2.6 \pm$ $0.5 \mathrm{mmol} / \mathrm{L}$.

Figure 2 shows a typical example of the changes in the concentrations of urea, glucose, and lactate in the dialysate during the infusion of 4 increasing concentrations of NA in a single catheter in one of the test subjects. There was considerable variability in the responses within a single drug concentration, particularly in the urea concentration. However, during the period of drug delivery, there was a consistent, gradual increase in the concentrations of urea and lactate, whereas the concentration of glucose decreased. At the start of each new drug concentration, flushing of the 
catheter caused a marked increase in urea. The first sample with each new drug concentration was therefore discarded. After this, plateaus were usually reached within 30 minutes.
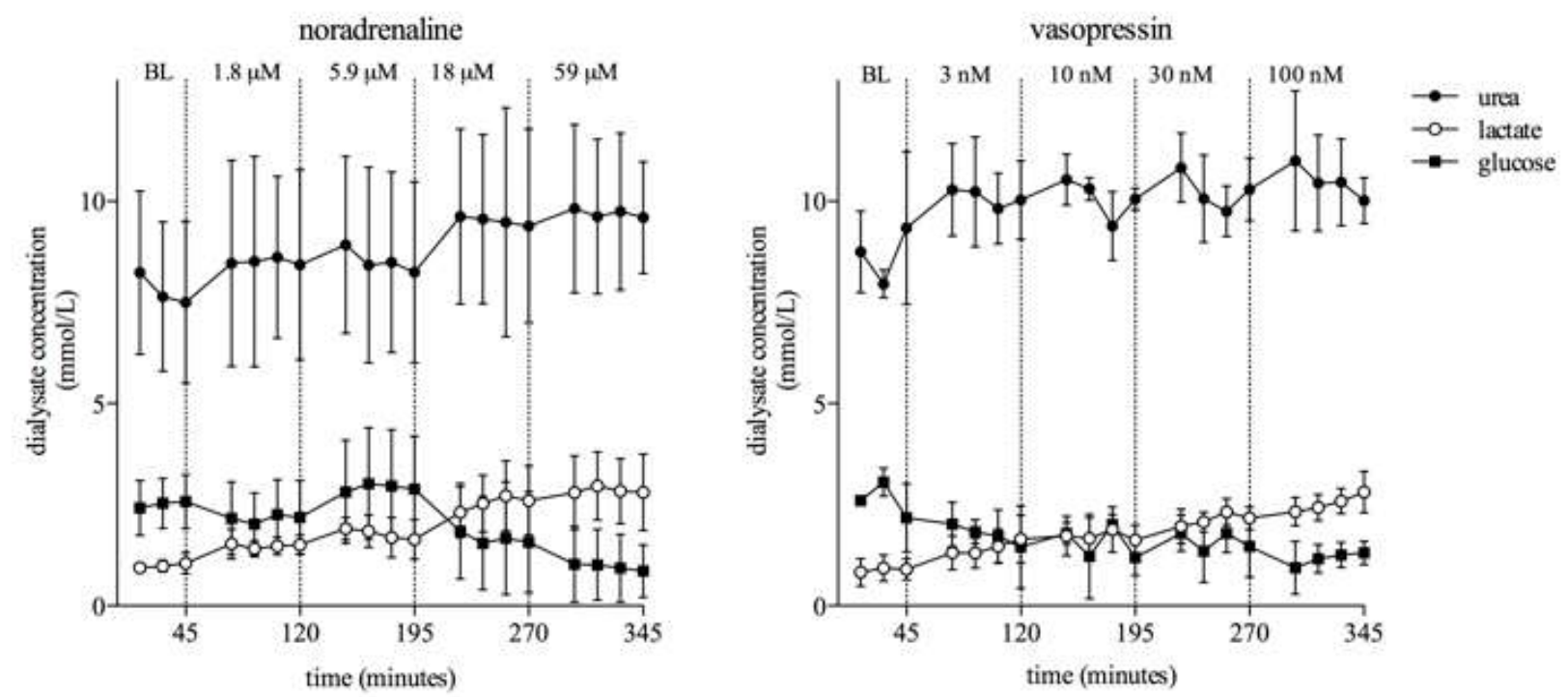

Figure 2. Dialysate concentrations (mmol/L) of urea, glucose, and lactate during microdialysis infusion of 4 increasing concentrations of noradrenaline. In most cases, plateaus were reached 30 minutes after the start of a new concentration with glucose and lactate, whereas the changes in the concentration of urea seemed to be affected by the flushing of the catheter at the start of each new drug concentration, in particular with vasopressin.

Changes in the concentrations of urea, glucose, and lactate in the dialysate, together with the fitted dose response curves from the $\mathrm{E}_{\max }$ model, are shown in Figures 3, 4, and 5, respectively. Table 1 gives a summary of the best-fit parameters for the different dose response curves. The $\mathrm{E}_{\max }$ model fitted well with the dose response data. Goodness-of-fit values were generally high (mean $\mathrm{R}^{2}$ $=0.91$, range $0.66-0.99)$ and $95 \%$ confidence intervals $(\mathrm{CI})$ were reasonably tight, particularly for $\mathrm{E}_{\max } . \mathrm{CI}$ for $\mathrm{ED}_{50}$ was wider for some curves because of the absence of plateaus. This was more common for VP, for which the range of concentrations was narrower. No significant differences were found between dose responses obtained using a single catheter and dose responses obtained using 4 different catheters. 
noradrenaline

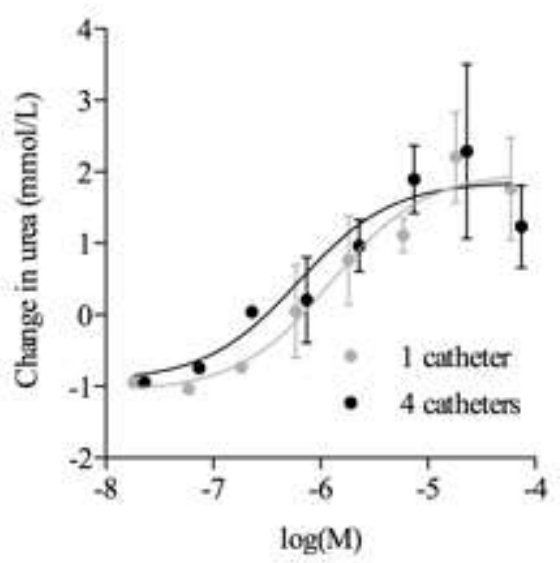

vasopressin

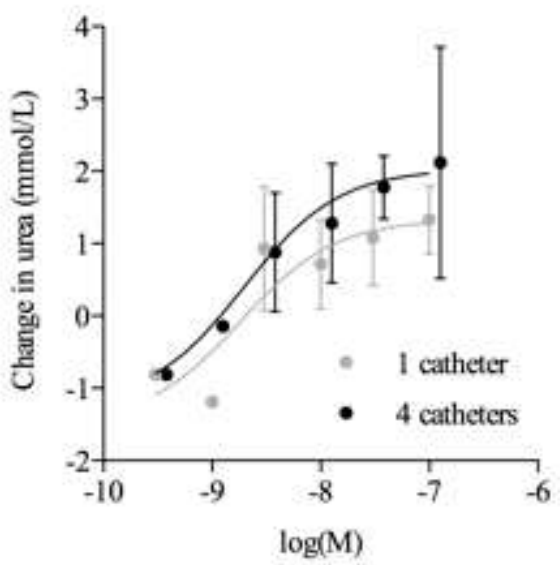

Figure 3. Absolute changes from baseline (mean $\pm S D$ ) of urea in the dialysate for increasing concentrations of noradrenaline (left panel, $n=5$ ) and vasopressin (right panel, $n=4$ ). There was a significant, dose-dependent increase in the urea concentration, but there was no difference between curves obtained from measurements in a single catheter and from measurements in four separate catheters.

noradrenaline

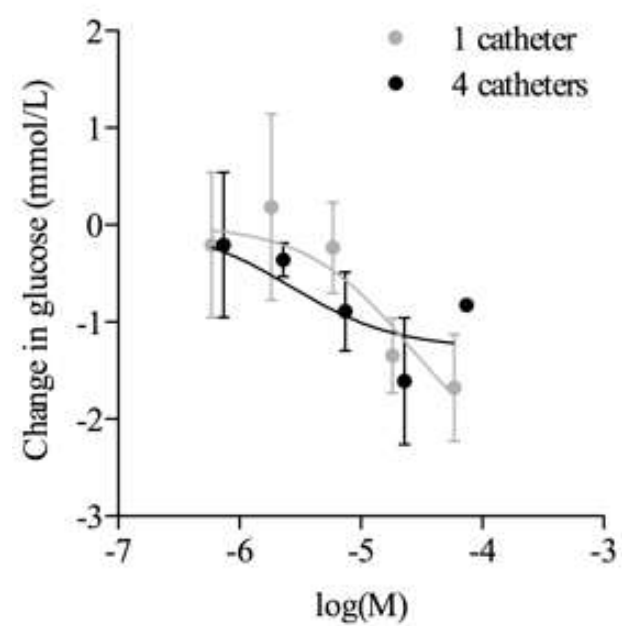

vasopressin

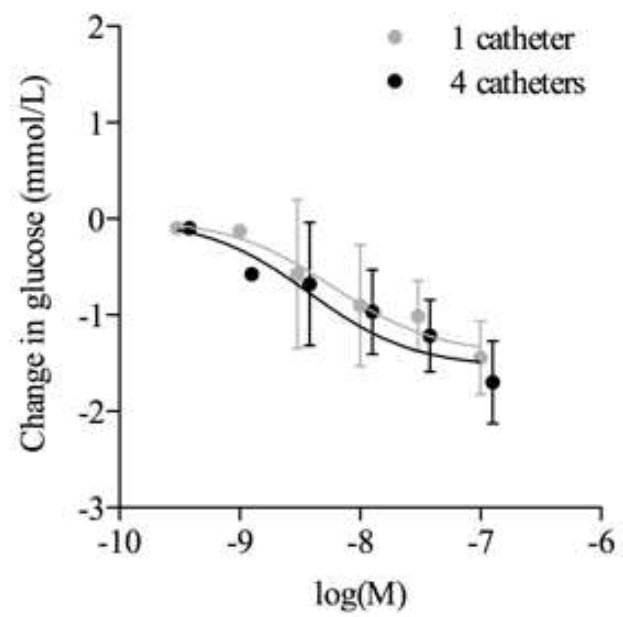

Figure 4. Absolute changes from baseline (mean \pm SD) of glucose in the dialysate for increasing concentrations of noradrenaline (left panel, $n=4$ ) and vasopressin (right panel, $n=4)$. There was a significant, dose-dependent reduction in the glucose concentration, but there were no differences between curves obtained from measurements in a single catheter and from measurements in four separate catheters. 


\section{noradrenaline}

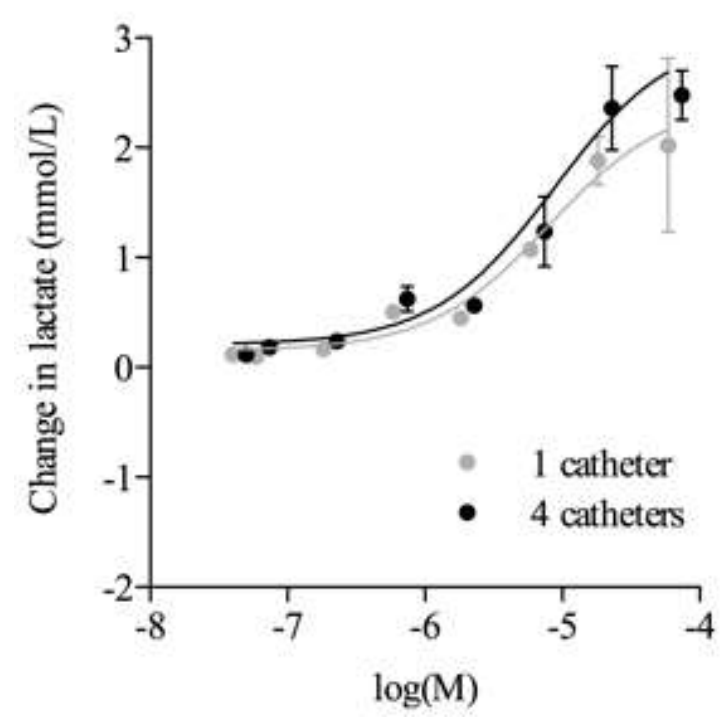

vasopressin

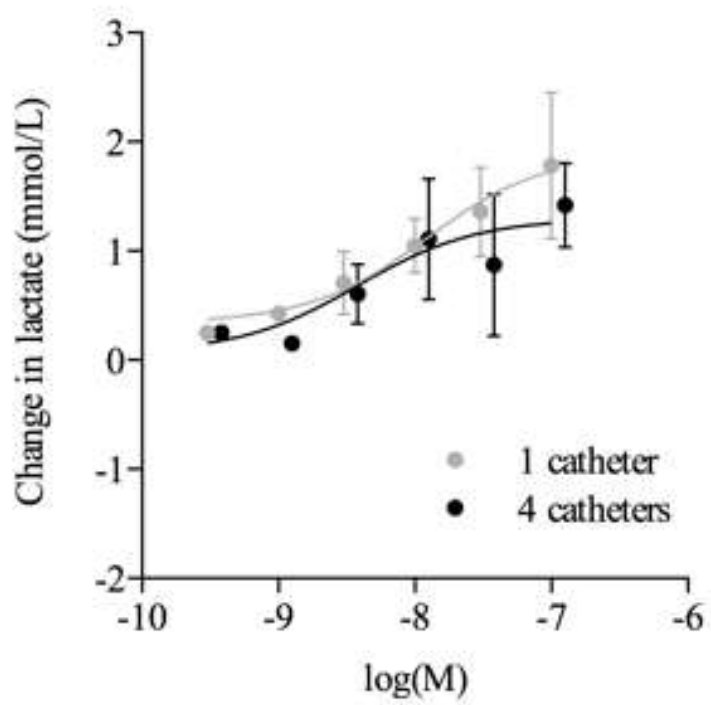

Figure 5. Absolute changes from baseline (mean $\pm S D$ ) of lactate in the dialysate for increasing concentrations of noradrenaline (left panel, $n=5$ ) and vasopressin (right panel, $n=5$ ). There was a significant, dose-dependent increase in the lactate concentration, but no difference between curves obtained from measurements in a single catheter or from measurements in four separate catheters. A significantly greater effect was observed in the change in lactate concentration with noradrenaline than with vasopressin.

Table 1. Summary of best-fit parameters (mean $\pm 95 \%$ CI) and goodness-of-fit values for the changes in urea, glucose, and lactate concentrations in the dialysate during microdialysis infusion of noradrenaline and vasopressin. $E_{\max }=$ maximum change from baseline, $E D_{50}=$ concentration resulting in $50 \%$ of $E_{\text {max }}, R^{2}=$ goodness-of-fit.

\begin{tabular}{|c|c|c|c|c|c|c|c|}
\hline & & \multicolumn{3}{|c|}{ Noradrenaline } & \multicolumn{3}{|c|}{ Vasopressin } \\
\hline & & $E_{\max }$ & $E D_{50}$ & $R^{2}$ & $E_{\max }$ & $E D_{50}$ & $R^{2}$ \\
\hline \multirow[t]{2}{*}{ Urea } & Single catheter & $3.1(2.4$ to 3.7$)$ & $-5.9(-6.3$ to -5.5$)$ & 0.97 & $2.9(0.091$ to 5.8$)$ & $-8.7(-10.5$ to -7.0$)$ & 0.82 \\
\hline & Multiple catheter. & $2.8(1.7$ to 3.8$)$ & $-6.2(-6.9$ to -5.5$)$ & 0.90 & $3.3(2.5$ to 4.1$)$ & $-8.7(-9.2$ to -8.3$)$ & 0.98 \\
\hline \multirow[t]{2}{*}{ Glucose } & Single catheter & $2.5(-1.4$ to 6.4$)$ & $-4.6(-5.9$ to -3.3$)$ & 0.89 & $1.4(0.87$ to 1.9$)$ & $-8.2(-8.6$ to -7.8$)$ & 0.96 \\
\hline & Multiple catheter. & $1.5(-3.6$ to 6.6$)$ & $-5.6(-7.1$ to -4.0$)$ & 0.65 & $1.5(0.65$ to 2.3$)$ & $-8.4(-9.0$ to -7.9$)$ & 0.90 \\
\hline \multirow[t]{2}{*}{ Lactate } & Single catheter & $2.2(1.7$ to 2.7$)$ & $-5.2(-5.6$ to -4.9$)$ & 0.80 & $1.6(1.2$ to 2.0$)$ & $-8.3(-8.7$ to -7.8$)$ & 0.63 \\
\hline & Multiple catheter. & $2.7(2.0$ to 3.4$)$ & $-5.2(-5.6$ to -4.8$)$ & 0.77 & $1.2(0.30$ to 2.2$)$ & $-8.5(-9.2$ to -7.8$)$ & 0.37 \\
\hline
\end{tabular}

\section{Discussion}

Microdialysis is a minimally invasive technique that offers interesting opportunities for studying the effects of vasoactive drugs on tissue blood flow in humans. While the infused drug concentrations may be high, the cumulated dose is extremely low and prevents systemic effects. The technique directly targets the extracellular space, which is where most drugs exert their pharmacological effects on extravascular structures rather than in the bloodstream itself (20). 
When a small, diffusible molecule is added to the microdialysis perfusate, its recovery is depends on the local blood flow in the tissue surrounding the catheter. This is because the local blood flow is responsible for a substantial part of the clearance of molecules out of the area of the catheter (5). This effect has been used in previous studies to assess blood flow, usually using ethanol (15) and, more recently, using urea (8-10). The recovery of urea increases significantly in response to a small reduction in blood flow and, because tissues other than the liver are not expected to produce or consume urea, this "urea clearance" method is sensitive and selective, which makes it suitable for measuring the effects of vasoactive drugs or drug candidates. An advantage of urea compared with ethanol for the assessment of blood flow is that the ethanol clearance method requires a perfusate velocity of more than $2 \mu \mathrm{l} /$ minute, which significantly decreases the recovery of other metabolites being studied in the same experiment (8).

Our new findings are that changes in urea clearance can be used as a measure of vasoconstriction in the skin on the delivery of increasing doses of vasoactive drugs through the microdialysis catheter. Pharmacodynamic characteristics based on the $\mathrm{E}_{\max }$ model could also be extrapolated from the data obtained, which made it possible to quantify the vasoactive effects of the drugs in terms of $\mathrm{ED}_{50}$ and $\mathrm{E}_{\max }$, both on local blood flow and on the tissue concentrations of the metabolic markers glucose and lactate.

An important difference with classical in vitro vessel preparations is that in this microdialysis model, the vessels and vascular effects can be investigated in a tissue environment that incorporates the influences not only of the vessels themselves, but also of nerve endings and mechanical and humoral factors (2). On the other hand, microdialysis studies tend to be lengthy, as very long stabilization times are sometimes needed. This may be problematic for some agonists, for which long infusions may lead to desensitization. Previously, without flushing catheters, we showed that it took up to 60 minutes for the urea concentrations to stabilise after the concentration of the vasoactive drug had been changed (8). In the present study, each of the four drug concentrations was infused during 75 minutes and always started with a 5-minute flush at a high perfusate flow 
rate, after which the dialysate concentrations of urea or metabolites stabilized quickly (the first sample after the flush was discarded). This supports the hypothesis that flushing the catheters can eliminate prolonged equilibrium times, thus making it possible to have shorter experimental protocols. However, it must be noted that this may not be applicable to other tissues, because the equilibrium time may vary depending tissue properties, type of drug, and the perfusate flow rate.

We expected that the dose response curves would differ depending on whether each concentration of drug was given in a separate catheter, or with increasing concentrations given through the same catheter. When increasing concentrations were given through a single catheter we expected the vascular responses to be affected by previous concentrations, as the vessels would already be partially constricted. We thought that this would result in less clearance of the drug and therefore more efficient uptake of drugs by the local vasculature (5). This effect would not be present when all concentrations were given at separate sites. However, we found no significant differences between a single-catheter or a multiple-catheter study design. Goodness-of-fit values were also comparable between the two designs, although they were more often lower for the multiple catheter design. This may be explained by the added variability as a result of the sampling at different skin sites (19). A single catheter design is obviously more practical and economical, as it requires only one catheter for each series of drug. On the other hand, using multiple catheters, together with a flush sequence, enables substantially shorter study protocols.

We did not measure the loss of drugs into the tissue. In a recent study by Ross et al. (22), a recovery of NA of $39 \%$ was obtained in human subcutaneous fat at a flow rate of $2 \mu \mathrm{l} / \mathrm{min}$ and a membrane cutoff of $20 \mathrm{kDa}$. We use a similar catheter $(20 \mathrm{kDa})$ at a flow rate of $0.5 \mu \mathrm{l} / \mathrm{min}$, which is expected to result in a higher delivery to tissue. Considering these findings, and based on unpublished data from our own previous experiments, we think it is safe to assume that the loss of NA into tissue in our study was almost $100 \%$. It should be noted, however, that it is very difficult to estimate the actual concentration within the catchment volume of the catheter., which is trivial in in 
vitro studies. Also, vasoactive drugs will probably affect their own clearance from the tissue by vasoconstriction of the blood vessels.

We found an $\mathrm{ED}_{50}$ of $10^{-6} \mathrm{~mol} / \mathrm{L}$ for NA and an $\mathrm{ED}_{50}$ of $10^{-8.7}$ for VP. These findings are in agreement with in vitro studies on human intestinal (25), umbilical (1), and uterine (17) arteries. Such an agreement in pharmacological parameters between in vitro and in vivo methods has not been found for other human in vivo skin models such as iontophoresis of acetylcholine and sodium nitroprusside (14), and reflects an advantage of the microdialysis model over more indirect routes such as iontophoresis, where the actual dose of drug in the target tissue is less certain.

To obtain more information about local blood flow and metabolism, we measured changes in the concentrations of the metabolic biomarkers glucose and lactate. A disadvantage when measuring glucose as a measure of local blood flow is that glucose uptake during acute ischaemia is energy-dependent (21). When NA was given, the increase in lactate in the tissue was higher than when VP was given, which confirms the well known metabolic effects of NA compared with VP (3).

Urea equilibrates more quickly than glucose and lactate, because the molecules are smaller $(4,7)$. The $\mathrm{ED}_{50}$ values of the responses of urea were lower than the $\mathrm{ED}_{50}$ values of the responses of glucose and lactate, although these differences were not significant. This points out another confounding factor to be considered when examining dose response effects: the temporal effect. It is exemplified in the results by the physiological delay in the increase in lactate compared with those in both urea and glucose.

At the lowest drug concentrations, we observed a decrease in urea in the dialysate. Some of these data points are from a single observation and should thus be interpreted with care. The most plausible explanation for a decrease in urea is the downward (although insignificant) trend in the concentration of urea after inserting the catheters. Despite a 60-minute recovery period after inserting the catheters, there may have been an ongoing trauma effect resulting in local vasodilatation. 
The changes in urea concentrations that we measured were substantial, $23 \%$ and $38 \%$ for NA and VP, respectively, and resulted in adequate dose response curves. At the same time, the absolute change from baseline was rather small, typically in the range of $2-3 \mathrm{mmol} / \mathrm{L}$ at the highest drug concentration. The absolute recovery of urea was between 8 and $11 \mathrm{mmol} / \mathrm{L}$, whereas the concentration in the perfusate was $20 \mathrm{mmol} / \mathrm{L}$. This is in agreement with our previous findings (8). The fact that urea concentrations varied so little and did not exceed $11 \mathrm{mmol} / \mathrm{L}$ could indicate that blood flow did not change substantially. However, this is unlikely, because the skin visibly blanched at the highest drug concentrations, and plateaus were obtained at the highest concentrations in most of the responses. Another explanation is that the sensitivity of the method is limited because of the high diffusibility of the molecules of urea in the skin. The molecules may diffuse over a larger area than those of the vasoactive drug, and so may partly include a volume of tissue where the vasoactive drugs have little or no effect. A possible improvement could be to change the microdialysis flow rate, although the effects on the sensitivity of the method are difficult to predict as the delivery of the drug would also be altered.

One important question is how do the drug responses in the skin compare with the corresponding effects in other tissues and vascular beds. Of course, they may vary among different drugs and the corresponding organs. However, the pharmacological site of action of both NA and VP is mainly the skin, and the model for these drugs therefore seems relevant.

\section{Conclusion}

This human in vivo vascular model offers interesting similarities to in vitro vascular models with respect to drug concentrations, dose-response characteristics, and data analysis. The results of this study indicate that urea clearance, and the analysis of metabolic markers glucose and lactate, can be used to characterize vascular dose-responses to vasoconstricting drugs delivered by microdialysis. The technique can be particularly useful in tissues where optical techniques cannot be applied. Further validation of the urea clearance technique and comparison with established optical methods 
are needed to get a better understanding for the sensitivity of the method in measuring changes in blood flow.

\section{Perspectives}

The results of this study indicate that in microdialysis studies, the clearance of urea from the catheter into the tissue, and the analysis of metabolic markers glucose and lactate, can be used to characterize vascular dose-responses to vasoconstricting drugs. The technique can be particularly useful in tissues where optical techniques cannot be applied.

Conflicts of interest: none.

\section{Acknowledgements}

This work was supported by Berzelius Clinical Research Center AB, Linköping, Sweden. The authors particularly thank Camilla Fredriksson and Johan Lindgren for their technical assistance. 


\section{References}

1. Altura BM, Malaviya D, Reich CF, and Orkin LR. Effects of vasoactive agents on isolated human umbilical arteries and veins. Am J Physiol 222: 345-355, 1972.

2. Angus JA, and Wright CE. Techniques to study the pharmacodynamics of isolated large and small blood vessels. J Pharmacol Toxicol Methods 44: 395-407, 2000.

3. Barth E, Albuszies G, Baumgart K, Matejovic M, Wachter U, Vogt J, Radermacher P, and Calzia E. Glucose metabolism and catecholamines. Crit Care Med 35: S508-518, 2007.

4. Baumeister FA, Rolinski B, Busch R, and Emmrich P. Glucose monitoring with long-term subcutaneous microdialysis in neonates. Pediatrics 108: 1187-1192, 2001.

5. Clough GF, Boutsiouki P, Church MK, and Michel CC. Effects of blood flow on the in vivo recovery of a small diffusible molecule by microdialysis in human skin. J Pharmacol Exp Ther 302: 681-686, 2002.

6. Cracowski JL, Gaillard-Bigot F, Cracowski C, Roustit M, and Millet C. Skin microdialysis coupled with Laser Speckle Contrast Imaging to assess microvascular reactivity. Microvasc Res 82: 333-338, 2011.

7. Ettinger SN, Poellmann CC, Wisniewski NA, Gaskin AA, Shoemaker JS, Poulson JM, Dewhirst MW, and Klitzman B. Urea as a recovery marker for quantitative assessment of tumor interstitial solutes with microdialysis. Cancer Res 61: 7964-7970, 2001.

8. Farnebo S, Samuelsson A, Henriksson J, Karlander LE, and Sjoberg F. Urea clearance: a new method to register local changes in blood flow in rat skeletal muscle based on microdialysis. Clin Physiol Funct Imaging 30: 57-63, 2010.

9. Farnebo S, Winbladh A, Zettersten EK, Sandstrom P, Gullstrand P, Samuelsson A, Theodorson E, and Sjoberg F. Urea clearance: a new technique based on microdialysis to assess liver blood flow studied in a pig model of ischemia/reperfusion. Eur Surg Res 45: 105-112, 2010. 
10. Farnebo S, Zettersten EK, Samuelsson A, Tesselaar E, and Sjoberg F. Assessment of blood flow changes in human skin by microdialysis urea clearance. Microcirculation 2010.

11. Forrest P. Vasopressin and shock. Anaesth Intensive Care 29: 463-472, 2001.

12. Gabrielsson JL, and Weiner DL. Methodology for pharmacokinetic/pharmacodynamic data analysis. Pharm Sci Technolo Today 2: 244-252, 1999.

13. Hammarlund-Udenaes M. The use of microdialysis in CNS drug delivery studies.

Pharmacokinetic perspectives and results with analgesics and antiepileptics. Adv Drug Deliv Rev 45: 283-294, 2000.

14. Henricson J, Tesselaar E, Persson K, Nilsson G, and Sjoberg F. Assessment of microvascular function by study of the dose-response effects of iontophoretically applied drugs (acetylcholine and sodium nitroprusside)--methods and comparison with in vitro studies. Microvasc Res 73: 143-149, 2007.

15. Henriksson J. Microdialysis of skeletal muscle at rest. Proc Nutr Soc 58: 919-923, 1999.

16. Hocht C, Opezzo JA, Bramuglia GF, and Taira CA. Application of microdialysis in clinical pharmacology. Curr Clin Pharmacol 1: 163-183, 2006.

17. Jovanovic A, Jovanovic S, Tulic I, and Grbovic L. Effect of oxytocin as a partial agonist at vasoconstrictor vasopressin receptors on the human isolated uterine artery. Br J Pharmacol 121: 1468-1474, 1997.

18. Karlander LE, Henriksson J, and Sjoberg F. Acidosis in muscle tissue distal to vascular contusion despite unchanged global blood flow in rats: an uncoupling of microvascular blood flow and metabolism? Microvasc Res 70: 111-115, 2005.

19. Kubli S, Waeber B, Dalle-Ave A, and Feihl F. Reproducibility of laser Doppler imaging of skin blood flow as a tool to assess endothelial function. J Cardiovasc Pharmacol 36: 640648, 2000.

20. Li Y, Peris J, Zhong L, and Derendorf H. Microdialysis as a tool in local pharmacodynamics. Aaps J 8: E222-235, 2006. 
21. Niklasson M, Holmang A, Sjostrand M, Strindberg L, and Lonnroth P. Muscle glucose uptake is effectively activated by ischemia in type 2 diabetic subjects. Diabetes 49: 1178$1185,2000$.

22. Ross HA, van Gurp PJ, Willemsen JJ, Lenders JW, Tack CJ, and Sweep FC. Transport within the interstitial space, rather than membrane permeability, determines norepinephrine recovery in microdialysis. J Pharmacol Exp Ther 319: 840-846, 2006.

23. Russell JA, Walley KR, Singer J, Gordon AC, Hebert PC, Cooper DJ, Holmes CL, Mehta S, Granton JT, Storms MM, Cook DJ, Presneill JJ, and Ayers D. Vasopressin versus norepinephrine infusion in patients with septic shock. N Engl J Med 358: 877-887, 2008.

24. Schmidt S, Banks R, Kumar V, Rand KH, and Derendorf H. Clinical microdialysis in skin and soft tissues: an update. J Clin Pharmacol 48: 351-364, 2008.

25. Vanner S, Jiang MM, Brooks VL, and Surprenant A. Characterization of vasopressin actions in isolated submucosal arterioles of the intestinal microcirculation. Circ Res 67: 1017-1026, 1990. 